NOTICE

PORTIONS OF THIS REPOST RRE ILIEGIBLE. I

LA- $-10078-$ IIS has been reproduced the broadest possible avail-

ability.

\title{
High-Efficiency Particulate Air Filter Behavior at High-Speed Flows
}

\author{
P. K. Tang \\ W. S. Gregory \\ C. I. Ricketts \\ P. R. Smith*
}

DISCLAIMER

\begin{abstract}
This report was prepared as an account of work sponsored by an agency of the United States Government. Neither the United States Government nor any agency thereof, nor any of their employees, makes any warranty, express or implied, or assumes any legal liability or responsibility for the accuracy, completeness, or usefulness of any information, apparatus, product, or process disclosed, or represents that its use would not infringe privately owned rights. Reference herein to any specific commercial product, process, or service by trade nane, tradenart, manufacturer, or otherwise does not necessarily constitute or imply its endoracment, recommendation, or favoring by the United States Government or any agency thereof. The view: and opinions of authors expressed herein do not necessarily state or reflect thowe of the United States Government or any agency thereof.
\end{abstract}
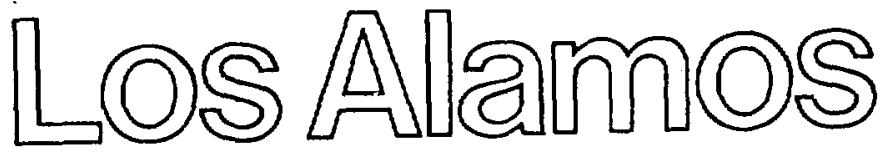

Los Alamos National Laboratory
Los Alamos,New Mexico 87545 
P. K. Tang, W. S. Gregory,

\author{
C. I. Ricketts, and P. R. Smith
}

\begin{abstract}
We will present a filter model based on the principle of fluid flow through porous media. The model includes both laminar and turbulent effects. The coefficients used in the model are determined empirically based on existing data. We

- also will discuss deviation from the model.
\end{abstract}

\title{
I. INTRODUCTION
}

High-efficiency particulate air (HEPA) filters used in the ventilation systems in the chemical and nuclear industries can be subjected to abnormally high flow conditions as a result of a tornado, explosion, or fire. Experimental evidence $^{1}$ indicates that the pressure drop across the HEPA filter increases nonlinearly at high-speed flow instead of linearly, which we generally expect in relatively low-speed flow regions for normal or near-normal conditions. ${ }^{2}$ We can take an entirely experimental approach toward determining all of the coefficients that influence filter and flow properties, or we can model the filter flow based on the principle of flow through porous media and determine the relationship between the flow rate and the pressure drop with most, if not ail, pertinent parameters explicitly included. Ėven so, some empirical coefficients still are needed. For practical purposes, we can combine some filter properties into these coefficients and determine them by experimental means. The number of 
coefficients with proper filter modeling is much less than the number of points used in a direct curve-segment method. We will review some theoretical work and then present a model that is suitable for our needs; some of the approach will be discussed, and finally, we will present our results.

\section{FILTER MODEL}

The pioneer work of $D^{\prime} A^{\prime} c y^{3}$ established the foundation for the principle of fluid flow through porous media. His experimental results indicate a linear reiationship between the flow rate and the pressure drop through an empirical constant, permeability. This parallels Hagen-Poiseille's conclusion of the fully developed laminar flow through a pipe quite wel1. ${ }^{4}$ It is not surprising that many theoretical models of flow through porous media are based on that concept (with different qualifications). The most successfut one among them is the Kozeny mode 1. 5 According to his theory, the porous medium is represented by an assemblage of channels of various cross sections and lengths. The flow through the channels is determined by the Navier-Stokes equations, and the permeability then is expressed in terms of viscosity and the porous medium properties. However, an empirical constant is needed to include the effect of the tortuous characteristic of the medium; a modification of the Kozeny model by Carman ${ }^{6}$ defines the constant, known as tortuosity, in a more explicit way. This new model still requires empirical coefficients to account for the uncertainty of determining various porous medium properties.

Another point of view on pressure drop with flow through a porous medium is based on the drag theory, and the dragging obstacles can be particles or fibers. A model ${ }^{7}$ using the fiber as a porous medium leads to a permeability that is weakly dependent on flow rate. Because of the complexity of the medium, some empirical adjustment is needed for this model.

So far, we have discussed the D'Arcy law and its derivatives, which are adequate only when the flow velocity is low; the pressure drop is proportional to the viscous dissipation by the porous medium. For channel flow, the dissipation mechanism changes from viscous to turbulent effects as the flow velocity increases, and the pressure drop is proportional to the kinetic energy of the stream. 4 Following the reasoning of Kozeny in modeling porous media as channels, a quadratic relation can be established between the pressure drop and the flow rate at high velocity. ${ }^{8}$ Again, an empirical coefficient equivalent to the resistance factor in pipe flow under turbulent conditions is introduced. The 
summation of viscous effects and turbulent dissipation leads to an equation proposed by Ergun. ${ }^{9}$

$$
\frac{\Delta p}{\ell}=150 \frac{(1-\varepsilon)^{2}}{\varepsilon^{3}} \frac{\mu u_{m}}{d_{p}^{2}}+1.75 \frac{(1-\varepsilon)}{\varepsilon^{3}} \frac{\rho u_{m}^{2}}{d_{p}}
$$

where

$$
\begin{aligned}
\Delta p & =\text { pressure drop, } \\
\ell & =\text { bed length, } \\
g & =\text { gravitational constant }, \\
\varepsilon & =\text { void fraction, } \\
\mu & =\text { viscosity, } \\
d_{p} & =\text { effective porous medium particle size, } \\
\rho & =\text { fluid density, and } \\
u_{m} & =\text { superficial velocity. }
\end{aligned}
$$

Superficial velocity is the flow velocity approaching the packed bed, not the average flow velocity in the interstitial region. Equation (1) is written in centimeter-gram seconds but also can be expressed in a different form,

$$
\Delta p=K_{L}+\frac{Q}{A^{3 / 2}}+K_{T^{p}} \frac{Q^{2}}{2 A^{2}},
$$

where $Q$ and $A$ represent volume flow rate and the frontal area of the packed column. It easily can be identified that

$$
u_{m}=\frac{Q}{A} \text {, }
$$




$$
K_{L}=150 \frac{(1-\varepsilon)^{2}}{\varepsilon^{3}} \frac{A^{1 / 2}}{d_{p}^{2}} \text {, }
$$

and

$$
K_{T}=3.5 \frac{(1-\varepsilon)}{\varepsilon^{3}} \frac{\ell}{d_{p}} \text {. }
$$

Clearly, $K_{L}$ and $K_{T}$ are dimensionless and are porous-medium dependent. Equation (2) is identical to Reynolds' expression ${ }^{10}$ on pipe flow in laminar and turbulent regions.

As we discussed earlier, no matter what theoretical model we choose, some empirical coefficients must be included to account for the complexity and uncertainty of the porous medium. Obviously, it does not matter if we obtain $K_{L}$ and $K_{T}$ first from Eqs. (4) and (5) and add experimental correction later or if we go ahead and determine the effective $K_{L}$ and $K_{T}$ directly from experiment. The task is not any more difficult than finding the correction factors alone because there are only two unknowns involved as presented in Eq. (2). From now on, we will use Eq. (2) as the foundation of our filter model regardless of what filtration media we use as long as we can determine the two coefficients through experimental or analytical means.

\section{TEST FACILITIES AND PROCEDURES}

Three major components can be used to produce proper flow conditions across HEPA filters. These are (1) an air compressor, (2) an air-storage volume, and (3) a test wind tunnel.

The Los Alamos National Laboratory has a $0.61-\mathrm{m}$ by $0.61-\mathrm{m}$ wind tunnel HEPA filter test facility, which is located at the New Mexico State University campus, Las Cruces, New Mexico. This apparatus is illustrated schematically in Fig. 1. The facility was designed to simulate tornado-induced flow transient conditions. Before a test, the compressor was used to pressurize the storage tanks with test air to an empirically determined level, typically no more than $2.4 \mathrm{MPa}$. The pressurized storage volume served as the test air source. The test air dryer prevented accumulation of liquid water in the test air storage volume. 


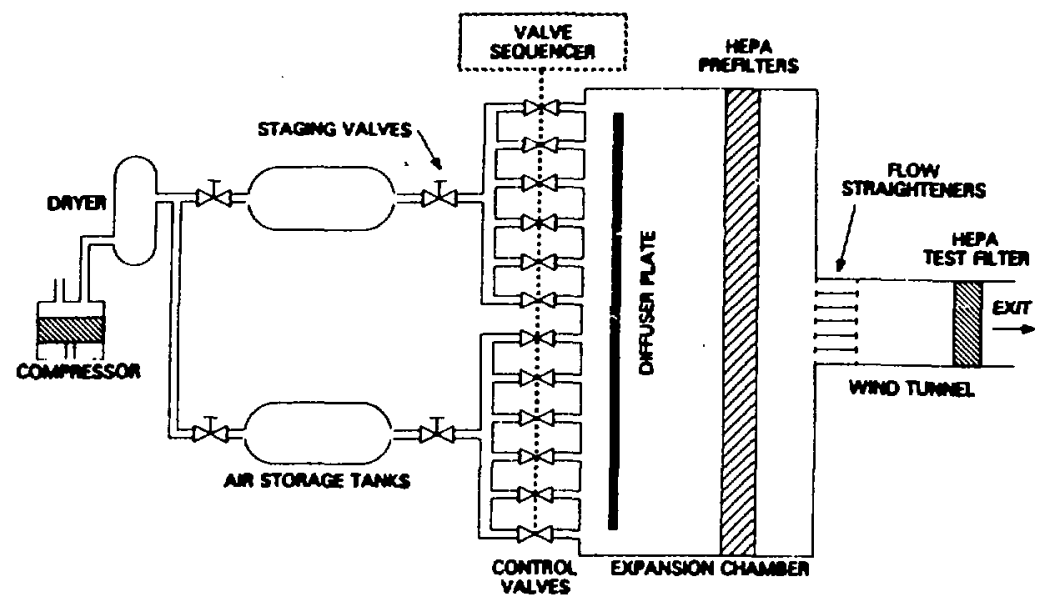

Fig. 1.

HEPA filter test facility schematic.

The test was initiated by the sequential opening of the solenoid control valves. The valve-sequence rate was in the range of 4 to 18 openings per second. The amount of time that the valves remained open was regulated manually or automatically by either of two preset variable mechanisms, a timer (1--6 s) or an event relay $(1--20 \mathrm{~s})$. Staging valve actuation allowed test air to flow from storage through intermediate piping to the solenoid valves, through sonically choked nozzles, and then into the expansion chamber. Air jets at the nozzle exits impinged on a diffuser plate and were broken up, thereby protecting the HEPA prefilters from damage. After leaving the pressurized expansion chamber, clean test air entered the wind tunnel, passed through flowstraightening tubes, and encountered the test filter from which the air was discharged to the atmosphere. The test pulse was terminated by closing the solenoid control valves using the valve sequencer.

To determine the relationship between pressure differential and a HEPA filter's flow rate for a simulated tornado, simultaneous measurement of one relativeiy stable and three transient test parameters was needed. The former, ambient-air barometric pressure, was recorded manually from a mercury-column barometer before the test. The latter included wind tunnel test air static pressure, dynamic pressure, and static temperature, which were measured at the duct cross-section center point just upstream of the test filter. Pitot-tube-sampled static and dynamic pressures were transferred by flexible tubing to individual variable-reluctance pressure transducers, which provided analog voltage input to 
two paper-chart-recorder channels. Test-air static temperature was monitored by a copper-constantan thermocouple connected to a third recorder channel. Each of two dual-channel thermal-pen recorders generated an individual chart record, thus providing documentation of three transient test-air parameters.

\section{RESULTS AND DISCUSSIONS}

Using the HEPH filter test data ${ }^{11}$ and the least-square fitting technique, we were able to find the laminar and turbulent coefficients for the filters listed in Table I. The results shown in Table $I$ are by no means conclusive because only one filter was used for each filter type. The comparisons of experimental measurements and curve fittings are shown in Figs. 2--5. (The experimental results are aiways higher than the curve-fit results at the highpressure differential end.) We believe that this discrepency is because of some filter structural change, mainly the reduction of void fraction that the relation we used [Eq. (2)] cannot take into account. However, the dependencies of the laminar and turbulent coefficients are clearly shown in Eqs. (4) and (5), based on the Ergun formula.

\section{v. SUMMARY}

We have presented a nonlinear filter model to relate the pressure drop and flow through HEPA filter. This model has some theoretical basis, but the formulation includes two empirical coefficients. Therefore, we included some experimental results to demonstrate the adequacy of this approach. More extensive

\section{TABLE I}

\section{FILTER COEFFICIENTS}

\begin{tabular}{|c|c|c|c|}
\hline Filter Maker & $\begin{array}{c}\text { Nominal Design } \\
\text { Flow Rate } \\
\end{array}$ & $K_{L}$ & $\underline{K_{T}}$ \\
\hline American Air, 4 & $14.2 \mathrm{~m}^{3} / \mathrm{min}$ & $7.298 \times 10^{6}$ & 10.50 \\
\hline American Air, 5 & $28.3 \mathrm{~m}^{3} / \mathrm{min}$ & $2.830 \times 10^{6}$ & $\cdot 12.25$ \\
\hline Flanders, 5 & $50.9 \mathrm{~m}^{3} / \mathrm{min}$ & $3.310 \times 10^{6}$ & 18.91 \\
\hline LUWA, 5 & $50.9 \mathrm{~m}^{3} / \mathrm{min}$ & $1.456 \times 10^{6}$ & 11.11 \\
\hline
\end{tabular}




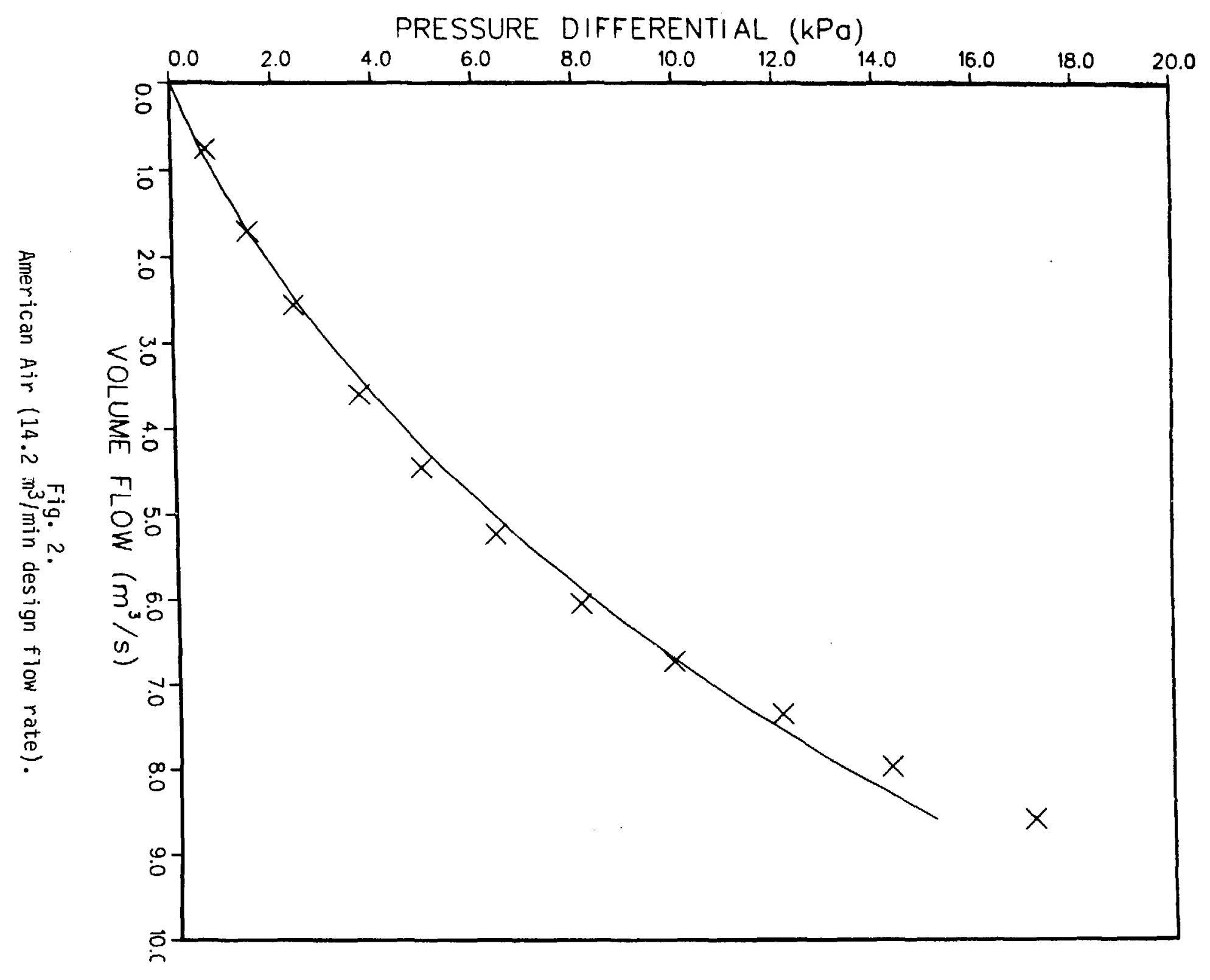




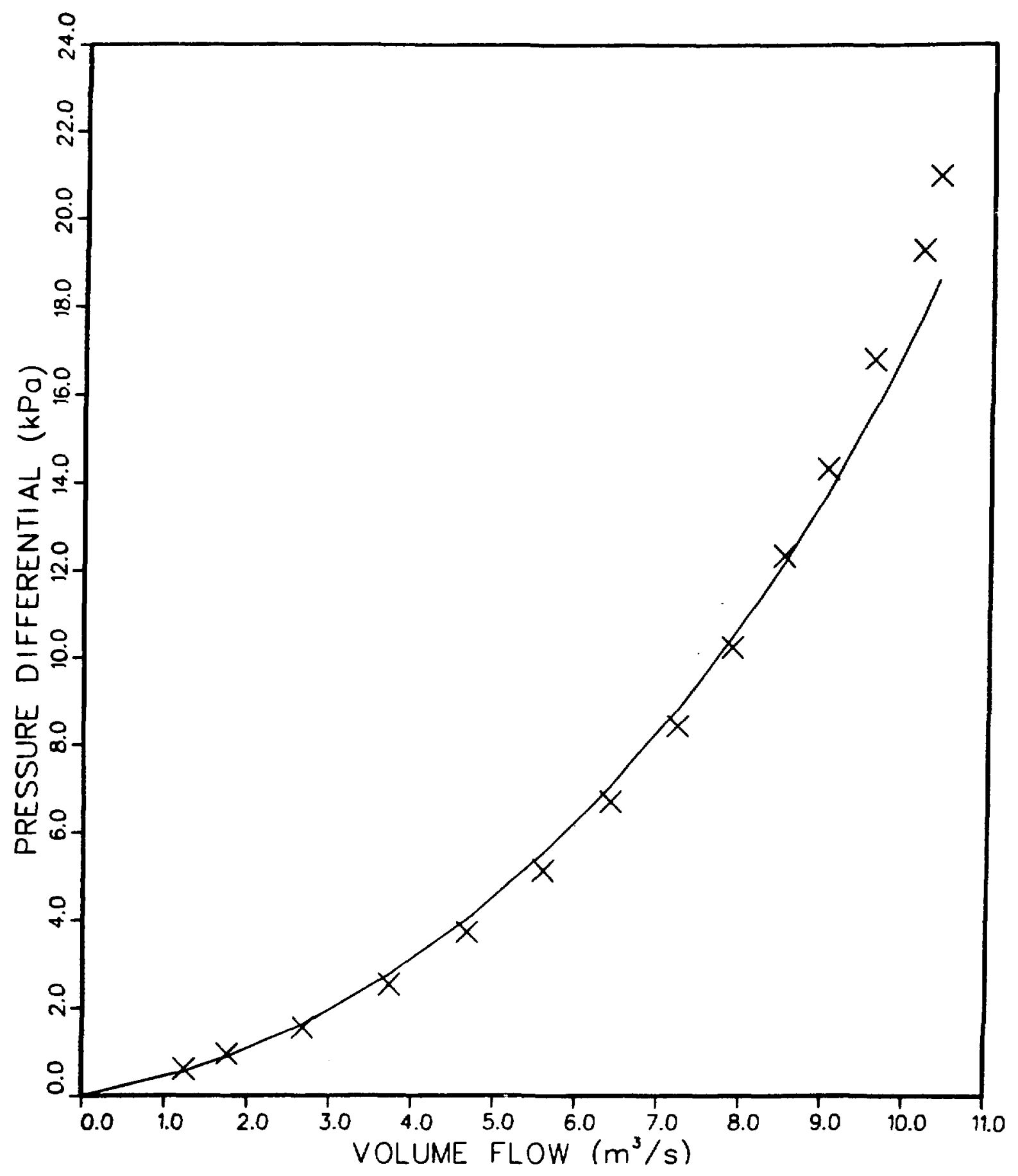

Fig. 3 .

American Air $\left(28.3 \mathrm{~m}^{3} / \mathrm{min}\right.$ design flow rate). 


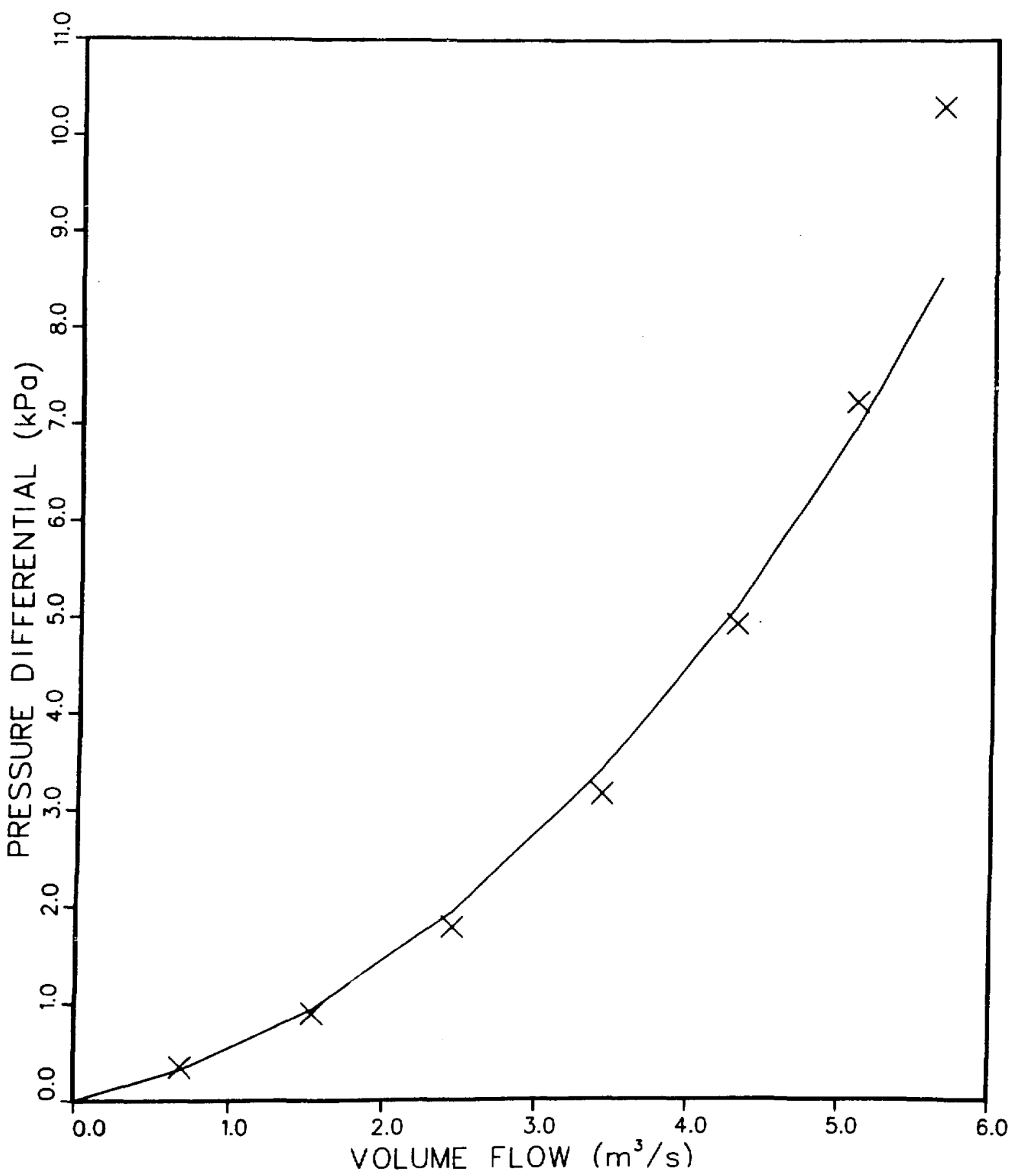

Fig. 4 .

Flanders $\left(50.9 \mathrm{~m}^{3} / \mathrm{min}\right.$ design flow rate). 


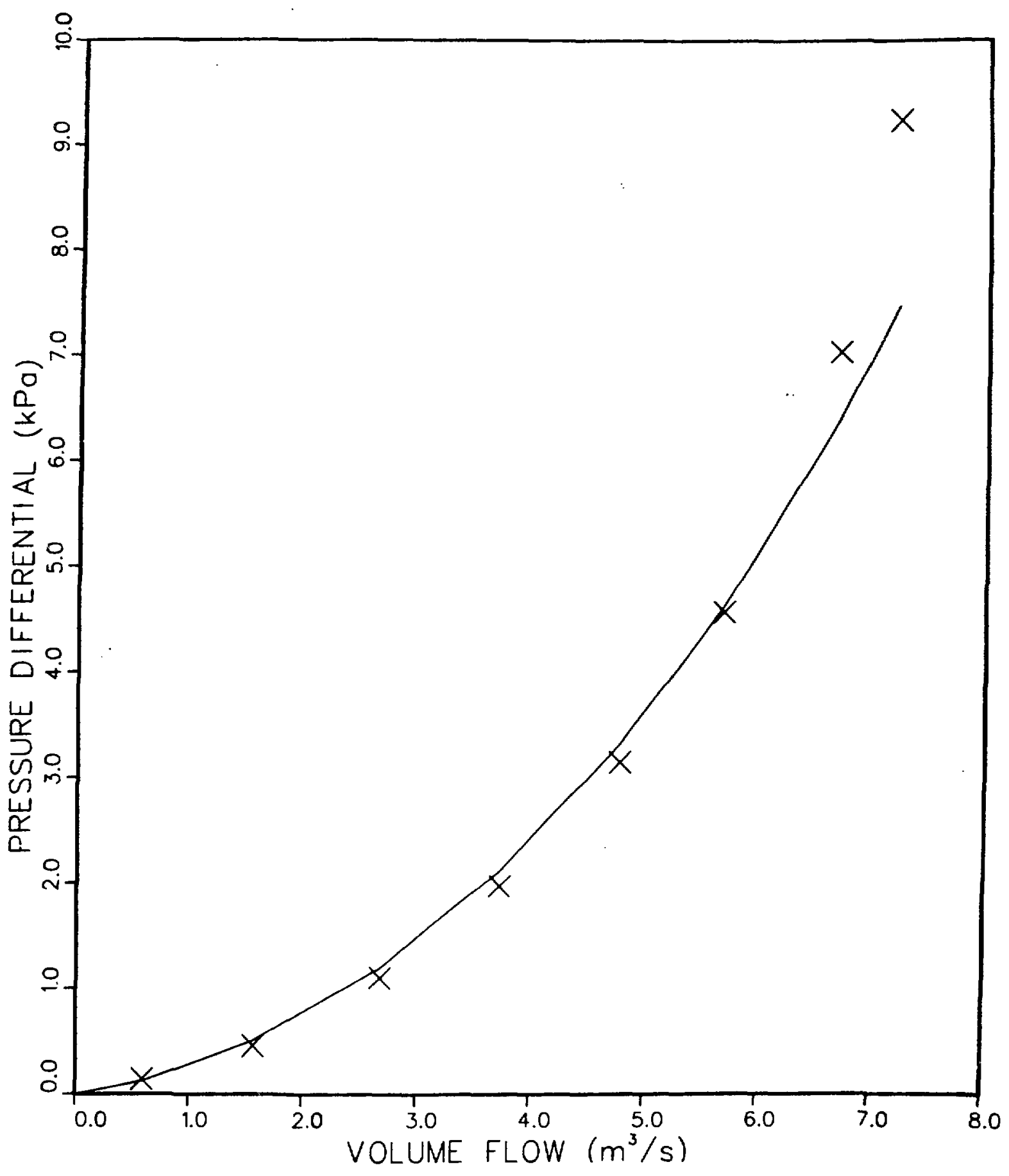

Fig. 5.

LUWA $\left(50.9 \mathrm{~m}^{3} / \mathrm{min}^{2}\right.$ design flow rate). 
experimental work should be carried out to form a reliable data base for more general applications. This model has been incorporated into a gas-dynamic transient computer code EVENT. 12

\section{REFERENCES}

1. W. S. Gregory, H. L. Horak, P. R. Smith, C. I. Ricketts and W. Gill, "Investigation of HEPA Filters Subjected to Tornado Pressure Pulses," Los Alamos Scientific Laboratory report LA-7202-MS (April 1978).

2. K. H. Duerre, R. H. Andrae, and W. S. Gregory, "TVENT, A Computer Program for Analysis of Tornado-Induced Transients in Ventilation Systems, "LoS Alamos Scientific Laboratory report 1.A-7397-M (July 1978).

3. H. D'Arcy, Les Fontaines Publiques de la Ville de Dijon (Victor Dalmont, Paris, France, 1856).

4. H. Schlichting, Boundary Layer Theory (McEraw-Hi11, New York, 1960), pp. 10-11 and 502--503.

5. J. Kozeny, "Uber kapillare Lejtung des Wassers in Boden," Akad. Wiss. Wien, Math. - naturw. Klasses, Sitzber. (Abt. IIa) 136, 271-306 (1927).

6. P. C. Carman, "Flow through Granular Beds," Transactions of the Institution of Chemical Engineers $\underline{15}, 150-166$ (1937).

7. A. E. Sheidegger, The Physics of Flow through Porous Media (University of Toronto Press, Toronto, 1972), pp. 146-148.

8. S. P. Burke and W. B. Plummer, "Gas Flow through Packed Columns," Industrial and Engineering Chemistry 20, 1196-1200 (1928).

9. S. Ergun, "Fluid Flow through Packed Columns," Chemical Engineering Progress 48, 89-94 (1952).

10. 0. Reynolds, Papers on Mechanical and Physical Subjects (Cambridge University Press, Cambridge, 1900).

11. C. I. Ricketts, "Tornado-Model Testing of HEPA Filters," Masters Thesis, Mechanical Engineering Department, New Mexico State University (December 1980). 
12. P. K. Tang, R. W Andrae, J. W. Bolstad, and W. S. Gregory, "EVENT User's Manual, A Computer Code for Analyzing Explosion-Induced Gas-Dynamic Transients in a Flow Network," Los Alamos National Laboratory report LA-9624-M (January 1983). 